Monte Carlo Implementation Of Up- Or Down-Scattering Due To Collisions With Material At Finite Temperature

S. Quaglioni, B. R. Beck

June 16, 2011 
This document was prepared as an account of work sponsored by an agency of the United States government. Neither the United States government nor Lawrence Livermore National Security, LLC, nor any of their employees makes any warranty, expressed or implied, or assumes any legal liability or responsibility for the accuracy, completeness, or usefulness of any information, apparatus, product, or process disclosed, or represents that its use would not infringe privately owned rights. Reference herein to any specific commercial product, process, or service by trade name, trademark, manufacturer, or otherwise does not necessarily constitute or imply its endorsement, recommendation, or favoring by the United States government or Lawrence Livermore National Security, LLC. The views and opinions of authors expressed herein do not necessarily state or reflect those of the United States government or Lawrence Livermore National Security, LLC, and shall not be used for advertising or product endorsement purposes.

This work performed under the auspices of the U.S. Department of Energy by Lawrence Livermore National Laboratory under Contract DE-AC52-07NA27344. 


\title{
Monte Carlo implementation of up- or down-scattering due to collisions with material at finite temperature
}

\author{
S. Quaglioni ${ }^{1}$ and B. R. Beck ${ }^{1}$ \\ ${ }^{1}$ Lawrence Livermore National Laboratory, Livermore, P.O. Box 808, California 94551
}

(Dated: June 15, 2011)

\begin{abstract}
The Monte Carlo All Particle Method generator and collision physics library features two models for allowing a particle to either up- or down-scatter due to collisions with material at finite temperature. The two models are presented and compared. Neutron interaction with matter through elastic collisions is used as testing case.
\end{abstract}

\section{INTRODUCTION}

The Monte Carlo All Particle Method (MCAPM) generator and collision library provides a means for client codes to access the All Particle Method (APM) data-base and simulate in-flight nuclear reaction physics. Here, "All" stands for the following finite list of particles that can be "followed" or "tracked" by the library: neutron, proton, deuteron, triton, ${ }^{3} \mathrm{He}$, alpha, and photon. All tracked particle types are coupled through the collision physics [1]. Provided an incident particle among those treated in the library, its initial energy, and the composition of the background material, the collision physics performed within the MCAPM library consists of three main parts: $i$ ) sampling the target isotope with which the collision will take place; $i i$ ) sampling the reaction that will occur for the sampled isotope; and $i i i$ ) sampling the characteristics (number, type, speed and direction) of the exiting particles produced by the sampled reaction. This sequence is appropriate for a background composition in which the target isotopes are at rest. However, in any real medium at a temperature above absolute zero the nuclei are in thermal agitation and possess a distribution of velocities. Therefore, the extra steps of sampling the velocity (speed and direction) of the target isotope at the time of collision and correcting data for its motion have to be taken.

To include the temperature of the target, the collision physics of point $i$ ) is performed using Dopplerbroadened (or heated) cross-section data [2]. The Doppler-broadened cross section in the laboratory reference frame for a projectile of kinetic energy $E$ is

$$
\sigma(E, T)=\frac{1}{v} \int_{\vec{V}_{t}} v_{r} \sigma\left(E_{r}, 0\right) M\left(\vec{V}_{t}, T\right) d \vec{V}_{t}
$$

Here $v=|\vec{v}|$ is the projectile speed, $\vec{V}_{t}$ is the target velocity, $v_{r}=\left|\vec{v}-\vec{V}_{t}\right|$ and $E_{r}$ are the relative speed and relative kinetic energy, respectively, $\sigma\left(E_{r}, 0\right)$ is the cold $(0 \mathrm{~K})$ cross section, and $M\left(\vec{V}_{t}, T\right)=(4 \pi)^{-1} M\left(V_{t}, T\right)$ is the normalized distribution of the target velocity, which we assume to be an isotropic, Maxwell-Boltzman distribution [3]:

$$
M\left(V_{t}, T\right)=\frac{4}{\sqrt{\pi}}\left(\frac{m_{t}}{2 T}\right)^{\frac{3}{2}} \exp \left(-\frac{m_{t} V_{t}^{2}}{2 T}\right) .
$$

We have used the notation $m_{t}$ to denote the target mass. Point-wise tabulated cross sections can be Doppler broadened exactly using the SIGMA1 kernel broadening method of Cullen and Weisbin [4]. Heated cross sections are pre-computed and stored for a fixed number of temperatures in the APM data-base generated by the MCFGEN package [5]. If the cross section in the data library is not processed to the temperature that is needed in the problem, MCAPM uses linear interpolation.

Once an isotope is selected from the background material, the collision library samples the velocity of the target at the time of collision. Given a projectile of velocity $\vec{v}$, the velocity $\vec{V}_{t}$ should be randomly chosen from the velocity distribution of target particles which collide with that projectile. The (conditional) probability for a collision with a target at velocity $\vec{V}_{t}$ in a time $\delta t$ is proportional to the probability of a collision, $\delta t \rho v_{r} \sigma\left(v_{r}, 0\right)$, times the probability that the target has velocity $\vec{V}_{t}, M\left(\vec{V}_{t}, T\right)$, for a resulting probability density

$$
P\left(\vec{v}, \vec{V}_{t}\right)=\frac{v_{r} \sigma\left(E_{r}, 0\right) M\left(\vec{V}_{t}, T\right)}{v \sigma(E, T)},
$$

where $\rho$ is the density of the selected target isotope and $\sigma(E, T)$ is the heated cross section of Eq. (1). The relative velocity $v_{r}$ and hence the cold cross section $\sigma\left(E_{r}, 0\right)$ are functions of $\vec{V}_{t}$. Therefore, sampling from Eq. (3) is very difficult, unless some approximations are introduced. Particularly, it is very hard to develop a sampling method that is both efficient and general enough to be applicable to a wide range of cross section shapes. In this report we describe the two models now available in MCAPM to sample the target velocity starting from Eq. (3). The first is the constant elastic cross section (CECS) model used in MCNP [6] (where it is known as free gas thermal model ) and THERMAL [7], which is implemented uniquely for neutron elastic scattering and based on the approximation that the elastic cold cross section is constant. A brief description of this model is given in Sec. II. The second model, applicable to all six projectiles of finite mass treated in MCAPM and to all types of reactions, replaces the continuum-energy cross section with a multi-group approximation and is presented in Sec. III. For both models, we describe how the motion of the target is taken into account when the kinematics of a collision are being calculated. The pro- 
jectile energy distributions produced by the two models are compared in Sec. IV, where we present, as a test case, neutrons interacting with matter through elastic collisions. Concluding remarks are shown in Sec. V.

\section{CONSTANT ELASTIC CROSS SECTION MODEL}

This model is similar to the thermal treatment known as the free-gas thermal model in MCNP [6], and is characterized by the following restrictions and assumptions:

1) Only neutron elastic scattering corrected: Thermal down- or up-scattering is only implemented for neutron as the incident particle when the neutron energy is comparable with the thermal energy of the target [8]. That is, when the neutron speed is much greater than the target thermal speed, thermal effects can be ignored. Only corrections to the elastic scattering data are performed. For all other cases the target is considered at rest.

2) Constant cross section: The neutron elastic cross section is assumed to be constant, $\sigma\left(E_{r}, 0\right)=$ $\sigma_{0}$, when sampling the velocity of the target. This is a good approximation for neutron elastic scattering on light nuclei, for which the cross section $\sigma\left(E_{r}, 0\right)$ at low energies is a nearly-constant function of $E_{r}$. For heavy nuclei, where $\sigma\left(E_{r}, 0\right)$ can vary rapidly at low energy, the approximation is justified if the moderating effect of scattering is small.

3) Free gas: It is assumed that intermolecular forces between target atoms can be ignored.

4) Maxwellian speed distribution for the target: The target is assumed to be a Maxwellian with temperature $T$, as defined in Eq. (2).

With these assumptions, the probability distribution for a target velocity of modulus $V_{t}$ and direction $\mu=$ $\cos (\vartheta)=\vec{V}_{t} \cdot \vec{v} /\left(V_{t} v\right)$ following from Eq. (3) is

$$
\begin{aligned}
P\left(V_{t}, \mu\right) & =C v_{r} M\left(V_{t}\right) V_{t}^{2}, \\
& =C \frac{v_{r}}{v+V_{T}}\left(v+V_{t}\right) M\left(V_{t}\right) V_{t}^{2},
\end{aligned}
$$

where $\mathrm{C}$ is a normalization constant and $v_{r}=\left(v^{2}+V_{t}^{2}-\right.$ $\left.2 \mu v V_{t}\right)^{1 / 2}$. The convenience of multiplying and dividing by $\left(v+V_{T}\right)$ will become apparent in the next paragraph.

The sampling of Eq. (4) is done using the rejection method described in Ref. [7], which we summarize here for completeness. The direction $\mu$ and the speed $V_{t}$ are sampled independently, as if they were uncorrelated. The direction $\mu$ is sampled from a uniform probability density function in the range $[-1,1]$, and the speed is sampled from the normalized envelope function

$$
\begin{aligned}
\mathcal{E}\left(V_{t}\right) & =\frac{\left(v+V_{t}\right) M\left(V_{t}\right) V_{t}^{2}}{v+\left(2 / \sqrt{\pi \alpha_{t}}\right)} \\
& =\frac{v}{v+\left(2 / \sqrt{\pi \alpha_{t}}\right)} P_{1}\left(V_{t}\right)+\frac{2 / \sqrt{\pi \alpha_{t}}}{v+\left(2 / \sqrt{\pi \alpha_{t}}\right)} P_{2}\left(V_{t}\right),
\end{aligned}
$$

where $\alpha_{t}=m_{t} / 2 T$, and $P_{1}\left(V_{t}\right)$ and $P_{2}\left(V_{t}\right)$ are the two normalized probability density functions given by:

$$
\begin{aligned}
& P_{1}\left(V_{t}\right)=M\left(V_{t}\right) V_{t}^{2}, \\
& P_{2}\left(V_{t}\right)=\frac{\sqrt{\pi \alpha_{t}}}{2} M\left(V_{t}\right) V_{t}^{3} .
\end{aligned}
$$

The rejection technique is implemented as follows. First, if $r_{1}<v /\left[v+\left(2 / \sqrt{\pi \alpha_{t}}\right)\right]$, where $r_{1}$ is a random deviate in the interval $[0,1), V_{t}$ is sampled from $P_{1}\left(V_{t}\right)$, else $V_{t}$ is sampled from $P_{2}\left(V_{t}\right)$. The sampled values of $\mu$ and $V_{t}$ are then accepted if $r_{2}<v_{r} /\left(v+V_{t}\right)$, where $r_{2}$ is a second random deviate in the interval $[0,1)$, else they are rejected and the procedure is repeated. Incidentally, we note that $v_{r} /\left(v+V_{t}\right) \leq 1$, by definition. The sampling of $V_{t}$ from $P_{1}$ is performed using the rejection method, with a comparison function suggested by Dermott E. Cullen [7]. Alternatively, the substitution $V_{t}=x / \sqrt{2 \alpha_{t}}$ reduces $P_{1}$ to sampling the distribution $\sqrt{2 / \pi} \exp \left(-x^{2} / 2\right) x^{2}$ for the variable $x$ as discussed in Appendix A. Defining $x=\alpha_{t} V_{t}^{2}$, the sampling of $P_{2}\left(V_{t}\right)$ reduces to sampling from the distribution $x \exp (-x)$.

The sampling of the target isotope and reaction are performed using heated cross section data. If the sampled reaction is elastic, then the target velocity is sampled as described above, and the sampling of the exiting particles' characteristics is implemented assuming that the cold elastic cross section is constant.

\section{A. Non-relativistic kinematics of an elastic collision}

In the CECS thermal model, which is restricted to elastic neutron-scattering data, the only outgoing particles are the neutron and the target. Assuming that the collision is isotropic in the center-of-mass (c.m.) frame, the angles of the neutron and target post-collision velocities with respect to the neutron initial velocity are obtained in a non-relativistic kinematics framework as follows.

In the c.m. frame, the velocities of the neutron and target before the collision are (with $m$ the mass of the neutron):

$$
\begin{gathered}
\vec{u}=\vec{v}-\vec{v}_{\mathrm{c} . \mathrm{m} .}=\frac{m_{r}}{m} \vec{v}_{r}, \\
\vec{U}_{t}=\vec{V}_{t}-\vec{v}_{\mathrm{c} . \mathrm{m} .}=-\frac{m_{r}}{m_{t}} \vec{v}_{r},
\end{gathered}
$$

where we have introduced the c.m. velocity $\vec{v}_{\text {c.m. }}$, the relative velocity $\vec{v}_{r}$, and the reduced mass $m_{r}=m m_{t} /(m+$ $m_{t}$ ) for the neutron-target system. We further define a system of Cartesian axis by choosing the $x$-axis to be 
aligned with the neutron's initial velocity in the laboratory frame, and the $y$-axis to be aligned with the component of the target velocity that is perpendicular to the incident-neutron velocity (i.e., $\vec{V}_{t}=\mu \hat{x}+\sqrt{1-\mu^{2}} \hat{y}$ ). In the c.m. frame, after the elastic collision, neutron and target speeds are the same as before the collision and only their directions change. The final velocities in the c.m. frame can be written as,

$$
\begin{aligned}
& \vec{u}^{\prime}=\vec{v}^{\prime}-\vec{v}_{\mathrm{c} . \mathrm{m} .}=\frac{m_{r}}{m} v_{r}(\alpha \hat{x}+\beta \hat{y}+\gamma \hat{z}), \\
& \vec{U}_{t}^{\prime}=\vec{V}_{t}^{\prime}-\vec{v}_{\mathrm{c} . \mathrm{m} .}=-\frac{m_{r}}{m_{t}} v_{r}(\alpha \hat{x}+\beta \hat{y}+\gamma \hat{z}),
\end{aligned}
$$

where $\vec{v}^{\prime}$ and $\vec{V}_{t}^{\prime}$ are the final velocities in the laboratory frame. For MCAPM, only the components of $\vec{v}^{\prime}$ and $\vec{V}_{t}^{\prime}$ parallel and perpendicular to $\vec{v}$ are needed. For these components, $\gamma$ only appears as $\gamma^{2}$ and it is removed via the identity $\alpha^{2}+\beta^{2}+\gamma^{2}=1$. Hence, only $\alpha$ and $\beta$ need to be sampled (their sampling is explained in Appendix B). Let $v_{\|}^{\prime}\left(V_{t \|}^{\prime}\right)$ and $v_{\perp}^{\prime}\left(V_{t \perp}^{\prime}\right)$ be the components of $\vec{v}^{\prime}$ $\left(\vec{V}_{t}^{\prime}\right)$ parallel and perpendicular to $\vec{v}$. After some math, the parallel components can be written as,

$$
\begin{aligned}
v_{\|}^{\prime} & =\frac{m v+m_{t}\left(\mu V_{t}+\alpha v_{r}\right)}{m+m_{t}}, \\
V_{t \|}^{\prime} & =\frac{m_{t} \mu V_{t}+m\left(v-\alpha v_{r}\right)}{m+m_{t}},
\end{aligned}
$$

and the perpendicular componets as,

$$
\begin{aligned}
v_{\perp}^{\prime} & =\frac{m_{t}\left[\left(1-\mu^{2}\right) V_{t}^{2}+\left(1-\alpha^{2}\right) v_{r}^{2}+2 \beta \sqrt{1-\mu^{2}} V_{t} v_{r}\right]^{1 / 2}}{m+m_{t}}, \\
V_{t \perp}^{\prime} & =\frac{\left[m_{t}^{2}\left(1-\mu^{2}\right) V_{t}^{2}+m^{2}\left(1-\alpha^{2}\right) v_{r}^{2}+2 m m_{t} \beta \sqrt{1-\mu^{2}} V_{t} v_{r}\right]^{1 / 2}}{m+m_{t}} .
\end{aligned}
$$

Equations (12)-(15) are derived for isotropic scattering in the c.m. frame. For non-isotropic elastic scattering, the only outgoing data present in nuclear data evaluations are the probabilities for scattering by an angle $\theta_{r}^{\prime}$ where $\theta_{r}^{\prime}$ is the angle between the projectile's velocity and the scattered neutron's velocity in the c.m. frame as seen in the relative frame. In this case, Eqs. (12)(15) are still valid provided we rotate $\alpha^{\prime}=\cos \left(\theta_{r}^{\prime}\right)$ and $\beta^{\prime}=\sqrt{1-\alpha^{\prime 2}} \cos \left(2 \pi r_{r}\right)$ in the relative frame to those in the c.m. frame. Here, $\alpha^{\prime}$ is sampled from the angular data and $r_{r}$ is a random deviate in the interval $[0,1)$. The rotation yields,

$$
\begin{aligned}
& \alpha=\cos \left(\theta_{r}\right) \alpha^{\prime}-\sin \left(\theta_{r}\right) \beta^{\prime} \\
& \beta=\sin \left(\theta_{r}\right) \alpha^{\prime}+\cos \left(\theta_{r}\right) \beta^{\prime}
\end{aligned}
$$

where $\theta_{r}$ is the angle between the projectile's velocity and the relative velocity (i.e., $\cos \left(\theta_{r}\right)=\left(v-\mu V_{t}\right) / v_{r}$ derived from $\vec{v} \cdot \vec{v}_{r}=\vec{v} \cdot\left(\vec{v}-\vec{V}_{t}\right)$.

\section{MULTI-GROUP CROSS SECTION MODEL}

This model is less restrictive than the CECS thermal treatment in that it can be applied to all kinds of reactions involving projectiles and targets of finite mass. Restrictions and assumptions are as follows:

1) Only reactions involving particles with finite mass are corrected: Thermal down- or up- scattering is implemented for six (neutron, proton, deuteron, triton, ${ }^{3} \mathrm{He}$, alpha) of the seven projectiles treated in MCAPM and for all targets of finite mass. Photon scattering data are left unchanged.

2) Multi-group cross section: We make the approximation of using the cross section $\sigma\left(E_{r}, 0\right)$ averaged with a particle spectrum $\Phi(E)$ over energy intervals or groups. The value of this multi-group cross section is taken to be constant over a group:

$$
\sigma_{g}^{0}=\frac{\int_{E_{g}}^{E_{g+1}} \sigma(E, 0) \Phi(E) d E}{\int_{E_{g}}^{E_{g+1}} \Phi(E) d E} .
$$

For this study, the energy flux is constant over each group, $\Phi(E)=1$.

3) Free gas: It is assumed that intermolecular forces between target atoms can be ignored.

4) Maxwellian speed distribution for the target: The velocity distribution of the target is assumed to be a Maxwellian with temperature $T$, as defined in Eq. (2).

In a medium at temperature $T$, the most probable speed for a target of mass $m_{t}$ is the thermal value of $V_{t}^{\mathrm{Th}}=\sqrt{2 T / m_{t}}$. Given an incident particle of velocity $\vec{v}$ traveling in the hot medium, for a sufficiently large $\beta$ the interval $I_{\beta}=\left[v-\beta V_{t}^{\mathrm{Th}}, v+\beta V_{t}^{\mathrm{Th}}\right]$ will contain 
the majority of the allowed values for the relative speed $v_{r}=\left|\vec{v}-\vec{V}_{t}\right|$. Following from Eq. (3), the probability distribution for the target velocity can then be written as:

$$
P\left(V_{t}, \mu\right)=C \frac{v_{r} \sigma\left(E_{r}, 0\right)}{q_{\max }} q_{\max } M\left(V_{t}\right) V_{t}^{2}
$$

where $C$ is a normalization constant and $q_{\max }$ represents the maximum of $v_{r} \sigma\left(E_{r}, 0\right)$ over the values of $v_{r}$ specified by the interval $I_{\beta}$,

$$
q_{\max }=\max _{v_{r} \in I_{\beta}} v_{r} \sigma\left(E_{r}, 0\right) .
$$

The quantity $v_{r} \sigma\left(E_{r}, 0\right) / q_{\max }$ is smaller than one for $v_{r}$ values in the interval $I_{\beta}$. Therefore, the target velocity can be sampled from Eq. (19) according to the following rejection technique. First, $V_{t}$ is sampled from the normalized envelope function

$$
\mathcal{E}\left(V_{t}\right)=M\left(V_{t}\right) V_{t}^{2}
$$

This can be done using the inversion method. Defining $x^{2}=2 \alpha_{t} V_{t}^{2}$ the sampling from $\mathcal{E}\left(V_{t}\right)$ reduces to sampling from the distribution $\sqrt{2 / \pi} \exp \left(-x^{2} / 2\right) x^{2}$ as discussed in Appendix A. Second, the target direction $\mu$ is sampled from a uniform probability density function in the range $[-1,1]$. Third, $v_{r}=\left(v^{2}+V_{t}^{2}-2 \mu v V_{t}\right)^{1 / 2}$ and $\sigma\left(E_{r}, 0\right)$ are calculated from the sampled values of $V_{t}$ and $\mu$. Finally, the sampled target speed and direction are rejected when $r q_{\max }>v_{r} \sigma\left(E_{r}, 0\right)$, where $r$ is a random deviate in the interval $[0,1)$.

The rejection method described above is easy to implement and has the advantage of being applicable to a wide range of cross section shapes. However, it can be quite inefficient if used with point-wise data, particularly for cross sections that present a sharp maximum for a very narrow interval of energies, in which case there is a high rate of rejection. In practice, we make the approximation of using the cross section averaged over energy intervals or groups introduced in Eq. (18). The value of this so-called multi-group cross section is taken to be constant over a group: The boundaries for the speed intervals corresponding to the energy groups are calculated ahead of time according to the relativistic expression $v_{g}=\sqrt{E_{g}\left(E_{g}+2 m\right) /\left(E_{g}+m\right)^{2}}$, where $m$ is the mass of the incident particle, and the constant $q_{\max }$ is obtained from

$$
q_{\max }=\max _{g \in\left[G_{\min }, G_{M a x}\right]} v_{g} \sigma_{g}^{0}
$$

where $G_{\min }$ and $G_{\max }$ are group boundaries such that $v_{G_{\text {min }}} \leq v-\beta V_{t}^{\mathrm{Th}}<v_{G_{\min }+1}$ and $v_{G_{M a x}} \leq v+\beta V_{t}^{\mathrm{Th}}<$ $v_{G_{\text {Max }}+1}$, respectively. Finally, target speed and direction are rejected when $r q_{\max }>v_{r} \sigma_{G}^{0}$, where $v_{r} \in$ $\left[v_{G}, v_{G+1}\right)$. For our tests we adopted $\beta=2$. The use of larger values of $\beta$ made no significant difference in the results.

\section{A. Non-relativistic kinematic transformations}

The MCAPM data are given in the rest frame of the target. Accordingly, in the multi-group cross section (MGCS) model we $i$ ) boost the velocity of the projectile to the target's rest frame, where we $i i$ ) sample the reaction and exiting particles' characteristics using the cold cross section and, finally, iii) transform the velocities of the collision products back to the laboratory frame. In this section we describe the kinematic transformations involved in steps $i$ ) and $i i i)$.

In the laboratory frame $(x y z)$ we take the $x$-axis to be along the projectile's velocity

$$
\vec{v}=v \hat{x}
$$

and the target's laboratory velocity to be in the $x$ - $y$ plane

$$
\vec{V}_{t}=V_{t}\left(\mu \hat{x}+\sqrt{1-\mu^{2}} \hat{y}\right)
$$

The boost described in $i$ ) changes the projectile's initial velocity. In the reference frame where the target is at rest $\left(x^{\prime} y^{\prime} z^{\prime}\right)$, the initial velocity of the projectile coincides with the relative velocity

$$
\vec{v}_{r}=v_{r} \hat{x}^{\prime}=\left(V_{t} \mu-v\right) \hat{x}+V_{t} \sqrt{1-\mu^{2}} \hat{y} .
$$

The collision physics of point $i i$ ) is performed by the collision routine of MCAPM, which returns the speed $u_{s}$ of the collision product, and its direction $\mu_{s}$ relative to the projectile's initial boosted velocity. The velocity of the collision product can be written as

$\vec{u}_{s}=u_{s}\left(\mu_{s} \hat{x}^{\prime}+\mu_{p} \sqrt{1-\mu_{s}^{2}} \hat{y}^{\prime}+\sqrt{1-\mu_{p}^{2}} \sqrt{1-\mu_{s}^{2}} \hat{z}^{\prime}\right)$,

where we have chosen the $y^{\prime}$ axis in the $x-y$ plane, and $\mu_{p}$ is a random number between -1 and 1 . The $x^{\prime} y^{\prime} z^{\prime}$ reference system is given by a rotation of $\theta_{r}=\arccos \left(\mu_{r}\right)$ degrees around the $z=z^{\prime}$ axis, where

$$
\mu_{r}=\frac{\vec{v}_{r} \cdot \hat{x}}{v_{r}}=\frac{V_{t} \mu-v}{\sqrt{V_{t}^{2}+v^{2}-2 \mu V_{t} v}} .
$$

To perform the final step, iii), we must first rotate the product's velocity through the negative of the angle $\theta_{r}$, which yields,

$$
\vec{v}_{s}^{\prime}=u_{s}\left(\begin{array}{ll}
\left(\mu_{r} \mu_{s}-\mu_{p} \sqrt{1-\mu_{r}^{2}} \sqrt{1-\mu_{s}^{2}}\right) & \hat{x} \\
\left(\sqrt{1-\mu_{r}^{2}} \mu_{s}+\mu_{r} \mu_{p} \sqrt{1-\mu_{s}^{2}}\right) & \hat{y} \\
\sqrt{1-\mu_{s}^{2}} \sqrt{1-\mu_{p}^{2}} & \hat{z}
\end{array}\right) .
$$

Finally, by adding the target's velocity to $\vec{v}_{s}^{\prime}$ we get the velocity of the collision products in the laboratory reference frame: 


$$
\vec{v}_{s}=\left(\begin{array}{ll}
{\left[\left(\mu_{r} \mu_{s}-\mu_{p} \sqrt{1-\mu_{r}^{2}} \sqrt{1-\mu_{s}^{2}}\right) u_{s}+v_{2} \mu_{t}\right]} & \hat{x} \\
{\left[\left(\sqrt{1-\mu_{r}^{2}} \mu_{s}+\mu_{r} \mu_{p} \sqrt{1-\mu_{s}^{2}}\right) u_{s}+v_{2} \sqrt{1-\mu_{t}^{2}}\right]} & \hat{y} \\
\sqrt{1-\mu_{s}^{2}} \sqrt{1-\mu_{p}^{2}} u_{s} & \hat{z}
\end{array}\right) .
$$

\section{NEUTRON INTERACTION WITH MATERIAL AT FINITE TEMPERATURE THROUGH ELASTIC COLLISIONS}

The two thermal scattering models described in Sec. II and III and the corresponding MCAPM routines have been tested by means of a Monte Carlo simulation in which an initial monochromatic source of fast neutrons interacts with matter and achieves thermal equilibrium through elastic collisions.

\section{A. Test}

Specifically, the simulation consisted in starting with a monochromatic source of $\left(N=5 \times 10^{5}\right)$ neutrons and letting it diffuse in a box containing thermal nuclei of a single isotope target at a temperature $T$. The Monte Carlo test code used for this simulation, relaxToMaxwellian, is part of the MCAPM package.

The program starts (at the time $t=0$ ) by setting the energy of each neutron to some initial value $E_{0}$ and calculating the corresponding speed $v_{0}$, characteristic time

$$
\tau_{0}=\frac{1}{\rho v_{0} \sigma\left(E_{0}, T\right)},
$$

and free-flight time $\vartheta_{0}$ (at the time $t=0$ ). Here $\sigma(E, T)$ is the hot elastic cross section for the chosen isotope. In general, the free-flight time is a random variable and can be determined by sampling it from the probability distribution $P(\vartheta)=\exp (-\vartheta / \tau) / \tau$ using the formula

$$
\vartheta=-\tau \ln (r)
$$

with $r$ a random number sampled from a uniform distribution in the interval $[0,1)$.

The program then follows the neutrons for a total of $10^{4}$ time steps. At each time step $i$, the program updates the time that each neutron can spend in free flight by subtracting the elapsed time interval $\delta t_{i}$. When the free-flight time of one of the neutrons becomes zero or negative, the program calls the MCAPM collision routine (i.e. the neutron undergoes a collision) and updates its energy $E_{i}$, speed $v_{i}$, characteristic time $\tau_{i}$, and freeflight time $\vartheta_{i}$. The time intervals $\delta t_{i}$ are chosen in such a way that thermalization is reached in about half of the total number of time steps independently of the target isotope chosen in the simulation. This is achieved by setting

$$
\delta t_{i}=0.002\left(\frac{m_{t}}{m}\right)^{0.8}\left\langle\tau_{i}\right\rangle
$$

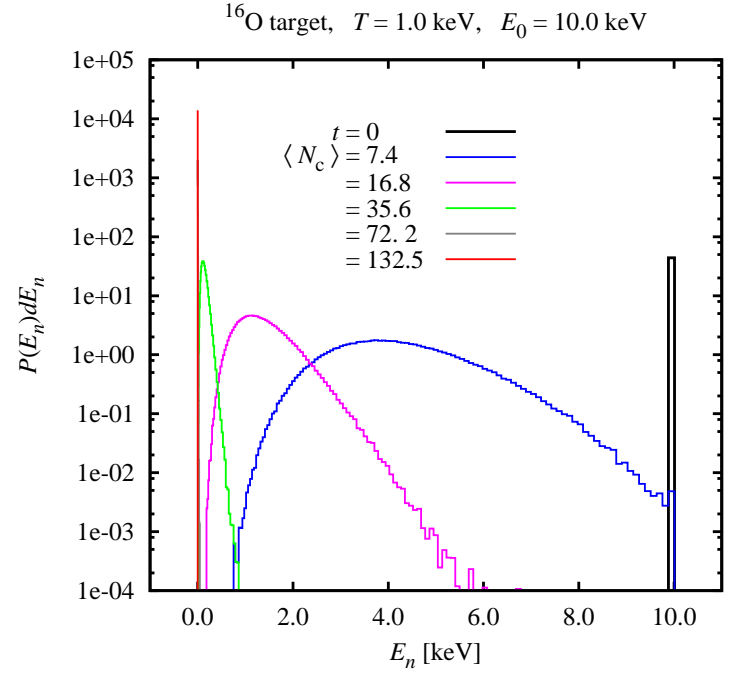

FIG. 1. Time-evolution, in terms of the average number of collisions $\left\langle N_{\mathrm{c}}\right\rangle$, of the neutron energy distribution for neutrons interacting with ${ }^{16} \mathrm{O}$ at $T=1.0 \mathrm{keV}$ through elastic scattering, when the motion of the target is ignored. The initial energy of the neutrons is $E_{0}=10.0 \mathrm{keV}$.

where $\left\langle\tau_{i}\right\rangle$ is the average characteristic time, 0.002 is an arbitrary small factor, $m$ and $m_{t}$ are neutron and target mass, respectively, and the "energy-exchange factor" $\left(m_{t} / m\right)^{0.8}$ is a good empirical fit to compensate for the fact that a projectile loses, on average, less energy per collision the heavier the target.

Finally, every 100 time steps, the program tallies the number of neutrons per energy interval and updates the neutron-energy distribution $P\left(E_{n}\right)$.

\section{B. Results}

In the scenario described in the above section, where only one kind of target isotope is considered and only elastic collisions are allowed, after a sufficient number of collisions the neutrons should thermalize and their energy distribution should relax to a Maxwell-Boltzmann distribution of temperature identical to that of the target nuclei. In our tests we considered three different isotopes, ${ }^{4} \mathrm{He},{ }^{16} \mathrm{O}$, and ${ }^{238} \mathrm{U}$.

We start by describing what happens in the simulation outlined above when the thermal motion of the target is ignored, and the target is considered at rest. As we will see, this can lead to serious error. Indeed, every time 
CECS: ${ }^{4} \mathrm{He}$ target, $T=0.1 \mathrm{keV}, \quad E_{0}=1.0 \mathrm{keV}$

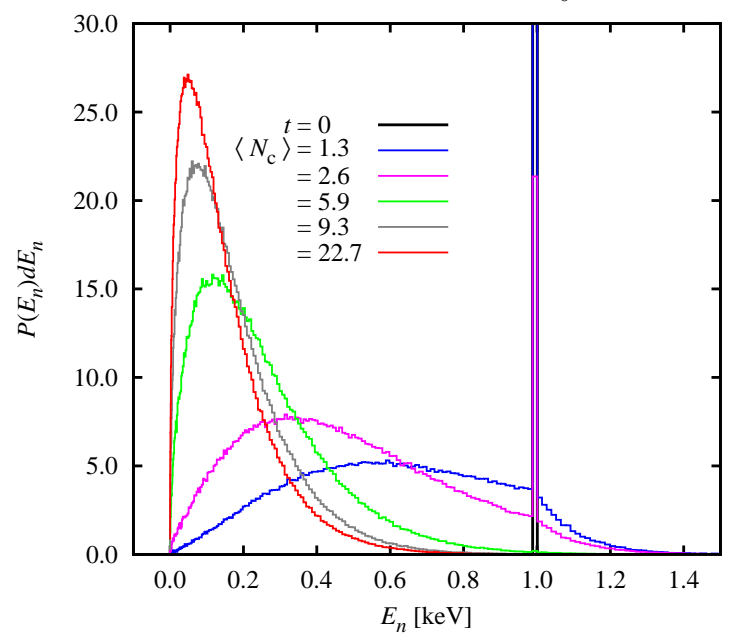

FIG. 2. Time evolution, in terms of the average number of collisions $\left\langle N_{\mathrm{c}}\right\rangle$, of the energy distribution for neutrons interacting with ${ }^{4} \mathrm{He}$ at $T=0.1 \mathrm{keV}$, obtained within the CECS thermal model. The neutrons have an initial energy of $E_{0}=1$ $\mathrm{keV}$.

a neutron performs an elastic collision with one of the target nuclei $\left({ }^{16} \mathrm{O}\right.$ in the example of Fig. 1), part of its energy is passed onto the target. In the situation shown in Fig. 1, in which the neutrons have an initial energy $E_{0}=10 \mathrm{keV}(t=0$ distribution $)$ and the ${ }^{16} \mathrm{O}$ targets a temperature $T=1 \mathrm{keV}$, at each elastic collision the neutrons always down-scatter and slowly cool down to zero energy, when in fact they should have relaxed to a Maxwell-Boltzmann distribution corresponding to the temperature of the background material.

As the time elapsed at each time step depends on the target's mass and neutron elastic cross section, in Fig. 1 and following we have chosen to label the time-evolution of the neutron-energy distribution by the average number of collisions, $\left\langle N_{\mathrm{c}}\right\rangle$. This allows for a more immediate comparison of the distributions we present.

Figure 2 shows the evolution with time of the energy distribution for neutrons interacting with ${ }^{4} \mathrm{He}$ at $T=0.1$ $\mathrm{keV}$, obtained within the CECS thermal model. The neutrons have an initial $(t=0) P\left(E_{n}\right)=\delta\left(E_{n}-E_{0}\right)$ energy distribution with $E_{0}=1 \mathrm{keV}$. At first the number of 1 $\mathrm{keV}$ neutrons gradually decreases, as they mostly downbut also up-scatter through the elastic collisions with the ${ }^{4} \mathrm{He}$ target. Already after about six collisions, the neutrons have an average energy of $\left\langle E_{n}\right\rangle=0.2 \mathrm{keV}$, and continue to cool down until they reach thermalization after about 23 collisions. As anticipated, for a light isotope such as ${ }^{4} \mathrm{He}$, the neutron elastic cross section is nearly constant at these low energies, and the CECS thermal scattering model is appropriate and leads to the correct neutron-energy distribution. Repeating this test within the MGCS model, we obtain equivalent results. This is to be expected, as the MGCS scattering model reduces

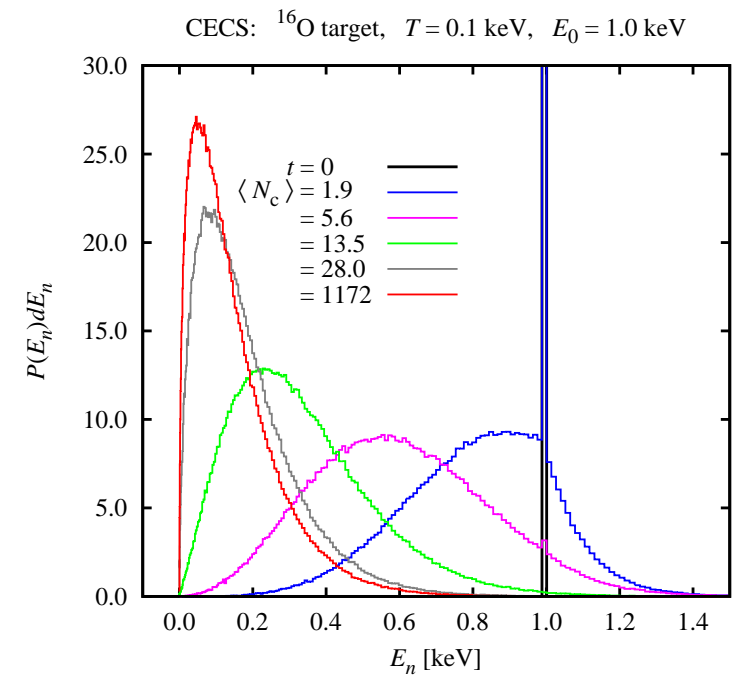

FIG. 3. Time evolution, in terms of the average number of collisions $\left\langle N_{\mathrm{c}}\right\rangle$, of the energy distribution for neutrons interacting with ${ }^{16} \mathrm{O}$ at $T=0.1 \mathrm{keV}$, obtained within the CECS thermal model. The neutrons have an initial energy of $E_{0}=1$ $\mathrm{keV}$.

to the CECS thermal model when the cold cross section is constant.

With ${ }^{16} \mathrm{O}$ we obtain analogous results, as shown in Fig. 3. Here, because of the larger mass of ${ }^{16} \mathrm{O}$, the amount of energy transferred from the neutrons to the target at each collision is smaller than in the ${ }^{4} \mathrm{He}$ case, and the neutrons are down-scattered at a slower pace. The average number of collisions required to reach thermalization is $\left\langle N_{\mathrm{c}}\right\rangle \approx 71.4$.

The ${ }^{4} \mathrm{He}$ and ${ }^{16} \mathrm{O}$ results obtained within the CECS thermal model are reexamined in Fig. 4 under a different perspective, and compared to calculations with a ${ }^{238} \mathrm{U}$ target isotope. This time, we present the behavior of the neutrons' average energy $\left\langle E_{n}\right\rangle$ as a function of the average number of collisions. As the energy of the target nuclei follows a Maxwell-Boltzmann distribution with $T=0.1 \mathrm{keV}$, in all three cases we expect that the average energy of the neutrons will reach the value $3 T / 2=0.15$ $\mathrm{keV}$ once thermalization is achieved. This is indeed the case for the light isotopes, whereas the neutrons scattering off ${ }^{238} \mathrm{U}$ come to the larger value of $\left\langle E_{n}\right\rangle=0.181(1)$ $\mathrm{keV}$.

The reason for this error can be quickly found in the plot of the $T \approx 0$ neutron elastic cross section for ${ }^{4} \mathrm{He},{ }^{16} \mathrm{O}$, and ${ }^{238} \mathrm{U}$, presented in the bottom panel of Fig. 4. While the assumption of constant cross section for $E_{n} \simeq T$ (which the CECS thermal model relies upon) is very good in the first two cases, clearly it is far from being valid for ${ }^{238} \mathrm{U}$, for which the neutron elastic cross section presents several narrow resonances in the energy range of interest. As a result, in a ${ }^{238} \mathrm{U}$ medium the neutron energy distribution does not relax to a 

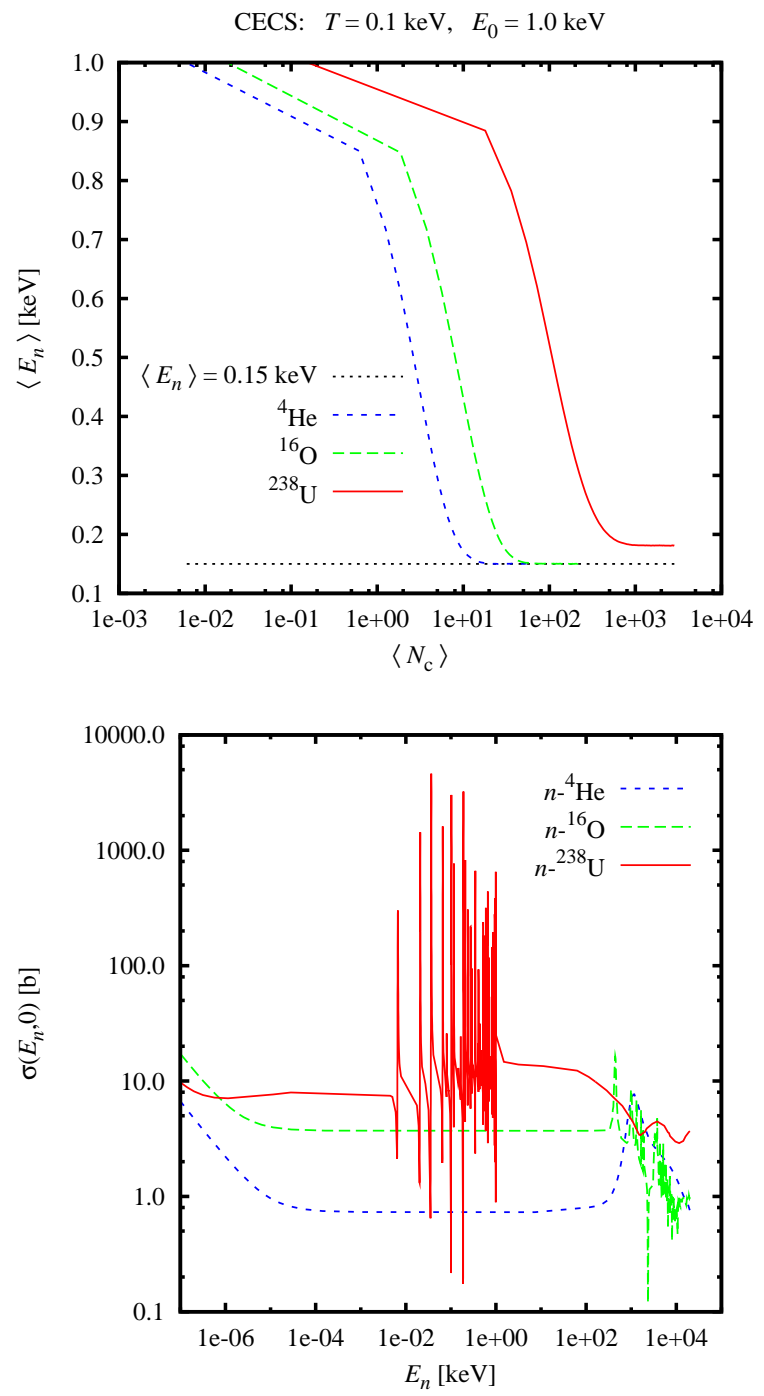

FIG. 4. Top panel: Average energy $\left\langle E_{n}\right\rangle$ as a function of the average number of collisions $\left\langle N_{c}\right\rangle$, for neutrons interacting with ${ }^{4} \mathrm{He}$ (short-dashed line), ${ }^{16} \mathrm{O}$ (long-dashed line) and ${ }^{238} \mathrm{U}$ (solid line) at $T=0.1 \mathrm{keV}$, obtained within the CECS thermal model. The neutrons have an initial energy of $E_{0}=1 \mathrm{keV}$. Bottom panel: $T \approx 0$ elastic cross section for $n-{ }^{4} \mathrm{He}$ (shortdashed line), $n-{ }^{16} \mathrm{O}$ (long-dashed line), and $n-{ }^{238} \mathrm{U}$ (solid line) elastic scattering. In the low-energy limit the elastic cross section is not constant and assumes a $1 / v$ behavior (with $v$ the neutron speed) because it has been heated to (the very small, but finite) room temperature, which is the lowest temperature in the MCAPM library.

Maxwellian, but rather to a widely oscillating function, as shown in the upper panel of Fig. 5.

To understand the origin of the oscillations observed in the top panel of Fig. 5, in the bottom panel we compare the $\left\langle N_{c}\right\rangle=1166$ distribution to the $T=0.1 \mathrm{keV} n_{-}{ }^{238} \mathrm{U}$ elastic cross section: peaks in the cross section correspond to dips in the distribution, and vice versa. This
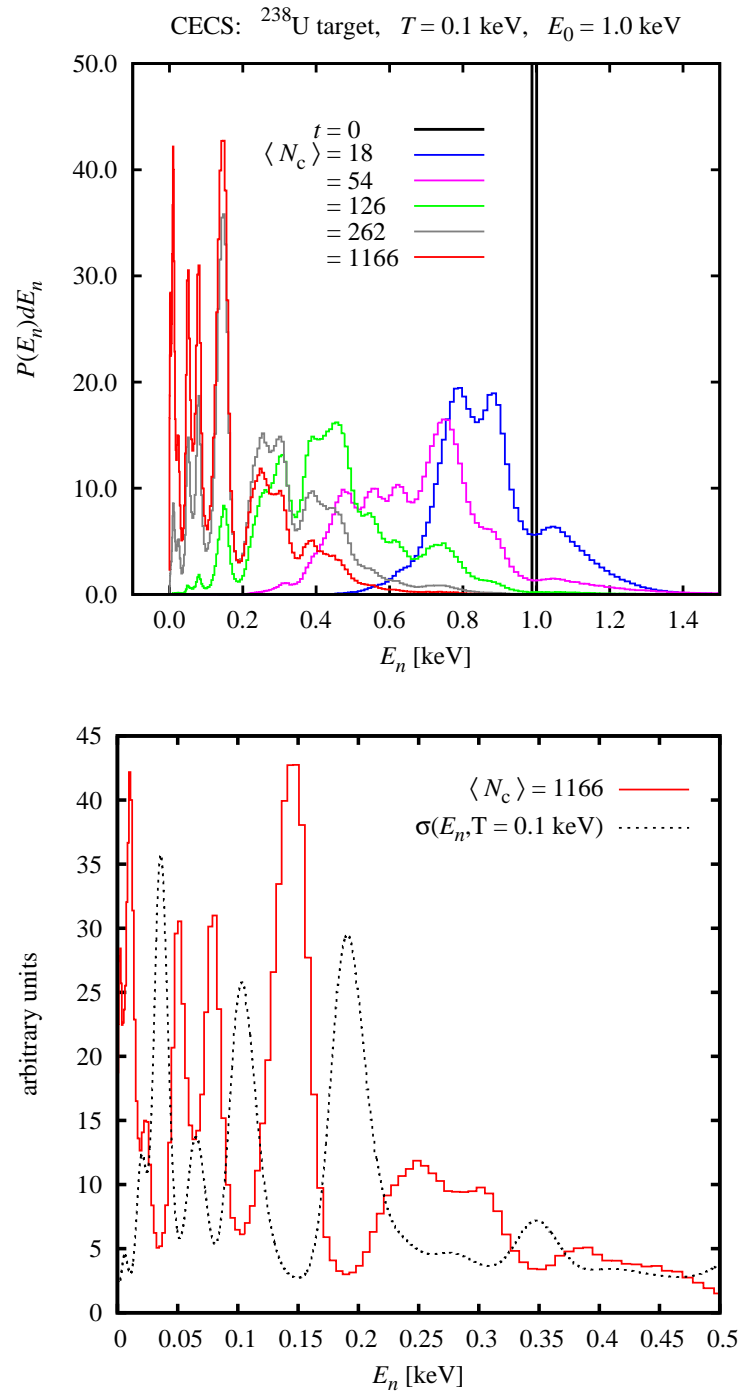

FIG. 5. Top panel: Time evolution, in terms of the average number of collisions $\left\langle N_{\mathrm{c}}\right\rangle$, of the energy distribution for neutrons interacting with ${ }^{238} \mathrm{U}$ at $T=0.1 \mathrm{keV}$, obtained within the CECS thermal model. The neutrons have an initial energy of $E_{0}=1 \mathrm{keV}$. Bottom panel: The energy distribution after 1166 collisions compared to the $T=0.1 \mathrm{keV} n-{ }^{238} \mathrm{U}$ elastic cross section.

behavior is caused by an imbalance between the sampling of the free-flight time of the neutrons and that of the velocity of the target. Indeed, while the first is based on the cross section heated from the $T=0 n^{-238} \mathrm{U}$ rate of Fig. 4 (bottom panel) according to Eqs. (29) and (30), the sampling of the target velocity is performed within the CECS thermal scattering model, and assumes that the $T=0$ cross section is constant. The result of this inconsistency between heated and cold cross sections is that, whenever the heated cross section has a peak, neutrons are scattered at a faster rate than they are thermalized, giving a dip in the neutron energy distribution. Vice versa, when the heated cross section presents a dip, 

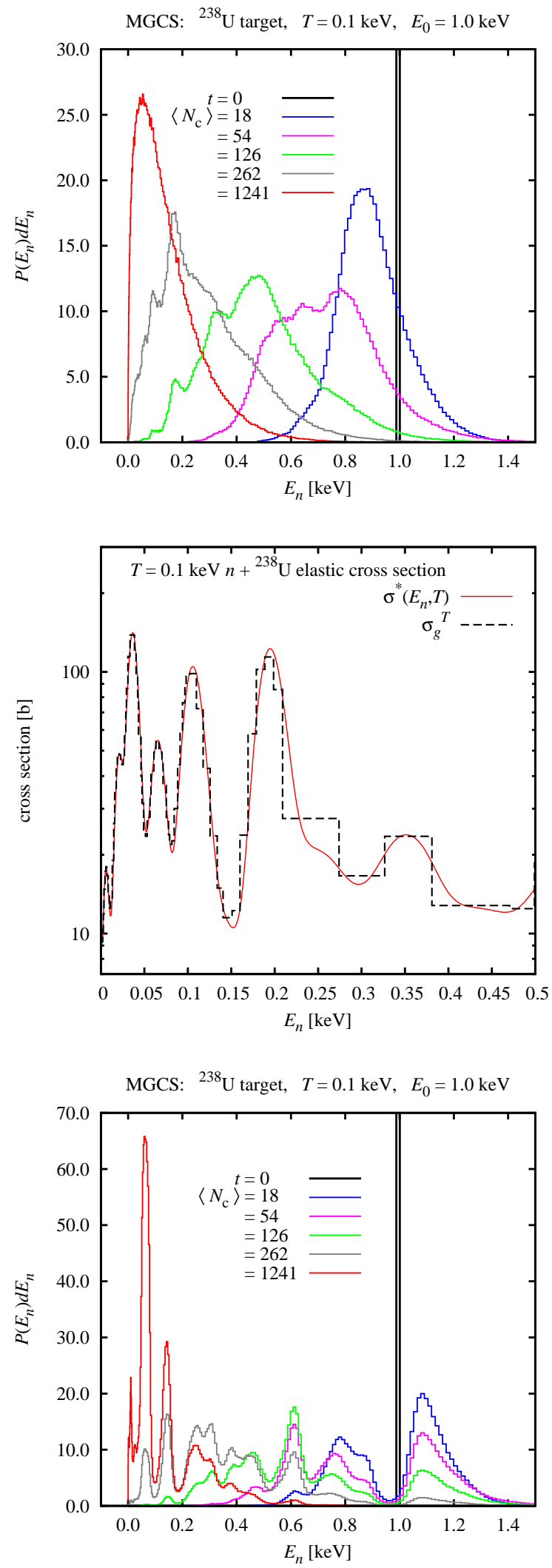

FIG. 6. Top and bottom panels: Same as in Fig. 5, but obtained within the MGCS thermal model using the heated cross section respectively as in Eqs. (35) and (33). Central panel: $T=0.1 \mathrm{keV} n-{ }^{238} \mathrm{U}$ elastic cross section of Eqs. (33) (dashed line) and Eq. (35) (solid line). the neutrons are not scattered fast enough and accumulate, creating a peak in the distribution. As we have seen from Fig. 4, this can lead not only to a neutron distribution that oscillates around a Maxwellian, but also to an incorrect average energy for the thermalized neutrons.

The MGCS thermal scattering model should offer a solution to the problem of low-energy cross sections with narrow resonances, as in the case of ${ }^{238} \mathrm{U}$. As anticipated earlier, the results of this model for light targets such as ${ }^{4} \mathrm{He}$ and ${ }^{16} \mathrm{O}$ (for which the low-energy cross section is a nearly-constant function of energy) are equivalent to those obtained within the CECS thermal model. The run-time for the simulations within the two different models is also comparable for these light isotopes.

The time-evolution of the energy distribution for an initial $1 \mathrm{keV}$ source of neutrons interacting with ${ }^{238} \mathrm{U}$ at $T=0.1 \mathrm{keV}$, obtained within MGCS model, is shown in the upper panel of Fig. 6. In this case, the neutrons do thermalize to a Maxwell-Boltzmann distribution in about 1241 collisions. As expected, the rate at which the neutrons loose energy through the collisions with the ${ }^{238} \mathrm{U}$ targets is about an order of magnitude smaller than for the much lighter ${ }^{16} \mathrm{O}$ (see Fig. 3). For cross sections that present narrow resonances in the energy region of interest, the MGCS model leads to a somewhat larger run time than that of the CECS thermal model, about three times larger for this specific simulation with ${ }^{238} \mathrm{U}$. This is caused by a higher rate of rejection during the sampling of the target velocity, as explained in Sec. III.

Caution has to be used in the implementation of the test described in Sec. IV A. In particular, to speed up the code, one could be tempted to make the approximation of using the multi-group cross section when calculating the characteristic time of the neutrons and set

$$
\tau_{i}=\frac{1}{\rho v_{i} \sigma_{g_{i}}^{T}}, \quad E_{g_{i}} \leq E_{i}<E_{g_{i}+1},
$$

with the multi-group heated cross section, calculated from point-wise data according to Eq. 1, given by (with $\Phi(E)$ the energy flux)

$$
\sigma_{g_{i}}^{T}=\frac{\int_{E_{g_{i}}}^{E_{g_{i}+1}} \sigma(E, T) \Phi(E) d E}{\int_{E_{g_{i}}}^{E_{g_{i}+1}} \Phi(E) d E} .
$$

In our simulations we used $\Phi(E)=1$. Having said that, the success of the simulation depends heavily on the consistency between the cross section used to sample the target velocity and that used to sample the free-flight time. In the MGCS model, the target velocity is sampled based on the $T=0$ multi-group cross section of Eq. (18), $\sigma_{g}^{0}$. Consequently, the correct characteristic time to achieve detailed balance, is given by

$$
\tau_{i}=\frac{1}{\rho v_{i} \sigma^{*}\left(E_{i}, T\right)}
$$

where $\sigma^{*}\left(E_{i}, T\right)$ is a "point-wise" cross section obtained 
from heating the $T=0$ multi-group cross section [9]:

$\sigma^{*}\left(E_{i}, T\right)=\frac{1}{v_{i}} \int_{\vec{V}_{t}} v_{r} \sum_{g} \sigma_{g}^{0} \chi_{\left[E_{g}, E_{g+1}\right)}\left(E_{r}\right) M\left(\vec{V}_{t}, T\right) d \vec{V}_{t}$,

where $v_{r}=\left|\vec{v}_{i}-\vec{V}_{t}\right|$, and $\chi_{\left[E_{g}, E_{g+1}\right)}\left(E_{r}\right)$ is the step function equal to one for $E_{g} \leq E_{r}<E_{g+1}$, and zero elsewhere,

$$
\chi_{\left[E_{g}, E_{g+1}\right)}\left(E_{r}\right)=\left\{\begin{array}{cc}
1 & \text { if } E_{r} \in\left[E_{g}, E_{g+1}\right) \\
0 & \text { elsewhere }
\end{array}\right.
$$

Although the differences between $\sigma_{g_{i}}^{T}$ and $\sigma^{*}\left(E_{i}, T\right)$ might seem minimal, as shown in the central panel of Fig. 6, the bottom panel of this same figure demonstrates the deleterious effects of choosing the former cross section over the latter when sampling the free-flight time of the neutrons in the case of a ${ }^{238} \mathrm{U}$ target: once again the neutron energy distribution relaxes to an oscillating function rather than to the expected Maxwell-Boltzmann distribution.

We note that this distinction between $\sigma_{g_{i}}^{T}$ and $\sigma^{*}\left(E_{i}, T\right)$ becomes important only when the $T=0$ cross section presents narrow resonances in the energy-region of interest. Indeed, for a nearly constant $T=0$ cross section $\sigma_{g_{i}}^{T} \approx \sigma^{*}\left(E_{i}, T\right)$.

Finally, for the tests within the MGCS model we used a modified APM data-library with $\sigma^{*}(E, T)$ in place of $\sigma(E, T)$. The modified data-base was generated with the program heatGroupXSec . py (Python based) that invokes the MCFGEN package [5] and is part of the MCAPM package.

\section{CONCLUSIONS}

In this report we reviewed the treatment of thermal scattering in MCAPM. First, we gave a brief description of the constant elastic cross section thermal model, which was ported from MCNP [6] (where it is known as free gas thermal model) and is based on the approximation that when sampling the target's velocity, the cold elastic cross section is treated as being constant. In MCAPM this model is implemented uniquely for neutron elastic scattering. Then, we presented the multi-group cross section model that replaces the continuum-energy cross section with a multi-group approximation. This second model is implemented for all six projectiles of finite mass treated in MCAPM and for all types of reactions. For both models, we described also how the motion of the target is taken into account when the kinematics of a collision are being calculated.

In the second part of the report we adopted the MCAPM collision routine to study the interaction of neutrons with matter at finite temperature through elastic collisions, and we compared the neutron energy distributions produced by the two thermal scattering models. Of the three target isotopes chosen for our simula- tions, the neutrons thermalize to a Maxwell-Boltzmann distribution using either of the thermal scattering models for the first two, ${ }^{4} \mathrm{He}$ and ${ }^{16} \mathrm{O}$. However, for ${ }^{238} \mathrm{U}$, the correct thermalization is achieved only using the MGCS model. Indeed, the $n+{ }^{238} \mathrm{U}$ elastic cross section at low energies, far from being nearly constant (an assumption of the CECS thermal model), presents several narrow resonances.

In general, the MGCS model is the safest thermal scattering model in MCAPM. In the cases in which the CECS thermal model is accurate, the MGCS model gives equivalent results in about the same amount of time. For heavy nuclei, where the $T=0$ cross section can vary rapidly at low energy, and the CECS thermal model can lead to serious error, the somewhat larger run time of the MGCS model is a small price to pay for achieving correct thermalization of the system.

Although it would be desirable to avoid the approximation of the continuum-energy cross section with a multi-group cross section, the MGCS thermal scattering model can become very inefficient if implemented with point-wise cross section data, particularly for cross sections that present a sharp maximum for a very narrow interval of energies, in which case there is a high rate of rejection in the sampling of the target speed.

Finally, caution has to be used when implementing the MGCS model into a thermalization program. Indeed, the success of the simulation depends heavily on the fulfillment of detailed balance. That is, the "right" cross section for the sampling of the free-flight time of the projectiles is that obtained by heating the $T=0$ cross section used for the sampling of the target velocity.

\section{ACKNOWLEDGMENTS}

We would like to thank G. Zimmermann and E. D. Brooks III for valuable discussions. Prepared by LLNL under contract No. DE-AC52-07NA27344.

Appendix A: Sampling from $(\sqrt{2 / \pi}) \exp \left(-x^{2} / 2\right) x^{2}$

We start by noting that

$$
\begin{aligned}
& \sqrt{\frac{2}{\pi}} \int_{0}^{x} e^{-\frac{1}{2} x^{\prime 2}} x^{\prime 2} d x^{\prime} \\
& =\frac{1}{(2 \pi)^{\frac{3}{2}}} \int_{0}^{x} e^{-\frac{1}{2} x^{\prime 2}} x^{\prime 2} d x^{\prime} \int_{0}^{\pi} \sin (\vartheta) d \vartheta \int_{0}^{2 \pi} d \phi \\
& =\frac{1}{(2 \pi)^{\frac{3}{2}}} \int_{0}^{x_{1}} \int_{0}^{x_{2}} \int_{0}^{x_{3}} e^{-\frac{1}{2}\left(x_{1}^{\prime 2}+x_{2}^{\prime 2}+x_{3}^{\prime 2}\right)} d x_{1}^{\prime} d x_{2}^{\prime} d x_{3}^{\prime} \\
& =I^{3}
\end{aligned}
$$

where the Cartesian variables $x_{1}^{\prime}, x_{2}^{\prime}$, and $x_{3}^{\prime}$ (integration limits $x_{1}, x_{2}$ and $x_{3}$ ) are given by the orthogonal trans- 
formation of the polar coordinates $x^{\prime}, \vartheta$, and $\phi(x, \vartheta$, and $\phi)$ :

$$
\begin{aligned}
& x_{1}^{\prime}=x^{\prime} \sin (\vartheta) \cos (\phi), \\
& x_{2}^{\prime}=x^{\prime} \sin (\vartheta) \sin (\phi), \\
& x_{3}^{\prime}=x^{\prime} \cos (\vartheta),
\end{aligned}
$$

and $I$ is the integral quantity:

$$
I=\frac{1}{\sqrt{2 \pi}} \int_{0}^{x_{1}} e^{-\frac{1}{2} x_{1}^{\prime 2}} d x_{1}^{\prime} .
$$

While inverting $I$ is not obvious, it is straight forward to invert $I^{2}$, by moving to the bi-dimensional polar coordinates $\rho^{\prime}$ and $\alpha^{\prime}$, with $x_{1}^{\prime}=\rho^{\prime} \cos \left(\alpha^{\prime}\right)$ and $x_{2}^{\prime}=\rho^{\prime} \sin \left(\alpha^{\prime}\right)$ (and integration limits $\rho=\sqrt{x_{1}^{2}+x_{2}^{2}}, \alpha=\arctan \frac{x_{2}}{x_{1}}$ ):

$$
\begin{aligned}
I^{2} & =\frac{1}{2 \pi} \int_{0}^{x_{1}} e^{-\frac{1}{2} x_{1}^{\prime 2}} d x_{1}^{\prime} \int_{0}^{x_{2}} e^{-\frac{1}{2} x_{2}^{\prime 2}} d x_{2}^{\prime} \\
& =\frac{1}{2 \pi} \int_{0}^{\rho} e^{-\frac{1}{2} \rho^{\prime 2}} \rho^{\prime} d \rho^{\prime} \int_{0}^{\alpha} d \alpha^{\prime} \\
& =\int_{0}^{y} e^{-y^{\prime}} d y^{\prime} \frac{1}{2 \pi} \int_{0}^{\alpha} d \alpha^{\prime}
\end{aligned}
$$

In Eq. (A9) we used the variable substitution $y^{\prime}=\rho^{\prime 2} / 2$ (and $y=\rho^{2} / 2$ ). Very easily now, we can use inversion techniques to sample the quantity $y$ from the distribution $e^{-y}$, and the angle $\alpha$ from a uniform probability density function in the range $[0,2 \pi]$. Given two random deviates, $r_{1}$ and $r_{\alpha}$ in the interval $[0,1)$ this sampling yields $y=$ $-\ln \left(r_{1}\right)$ and $\alpha=2 \pi r_{\alpha}$ and hence:

$$
\begin{aligned}
& x_{1}^{2}=-2 \ln \left(r_{1}\right) \cos ^{2}\left(2 \pi r_{\alpha}\right), \\
& x_{2}^{2}=-2 \ln \left(r_{1}\right) \sin ^{2}\left(2 \pi r_{\alpha}\right) .
\end{aligned}
$$

The third coordinate in Eq. (A2) can be sampled, once again, by choosing two more random deviates, $r_{2}$ and $r_{3}$ in the interval $[0,1)$ as $x_{3}^{2}=-2 \ln \left(r_{2}\right) \cos ^{2}\left(2 \pi r_{3}\right)$ [or, equivalently, $\left.x_{3}^{2}=-2 \ln \left(r_{2}\right) \sin ^{2}\left(2 \pi r_{3}\right)\right]$.

Overall, three independent random deviates, $r_{1}, r_{2}$, and $r_{3}$, are sufficient to sample $x$ from the distribution $(\sqrt{2 / \pi}) \exp \left(-x^{2} / 2\right) x^{2}$ :

$$
x=\sqrt{-2 \ln \left(r_{1}\right)-2 \ln \left(r_{2}\right) \cos ^{2}\left(2 \pi r_{3}\right)} .
$$

\section{Appendix B: Sampling isotropic direction in 3D}

The algorithm used to sample the quantities $\alpha$ and $\beta$ of Sec. II A is as follows. Given two random deviates $r_{x}$ and $r_{y}$ in the interval $[0,1)$, we generate the quantities $x$ and $y$ uniformly distributed in the interval $(-1,1)$ :

$$
\begin{aligned}
& x=1-2 r_{x}, \\
& y=1-2 r_{y} .
\end{aligned}
$$

We then calculate $\rho^{2}=x^{2}+y^{2}$, and reject and repeat the sampling if $\rho^{2}>1$. Next we calculate $z=\sqrt{1-\rho^{2}}$. Finally, the random quantities $\alpha$ and $\beta$ are given by:

$$
\begin{aligned}
& \alpha=2 x z, \\
& \beta=2 y z .
\end{aligned}
$$

This rejection technique is used in alternative to calculating

$$
\begin{aligned}
& \alpha=\sqrt{1-\mu^{2}} \cos (\phi), \\
& \beta=\sqrt{1-\mu^{2}} \sin (\phi),
\end{aligned}
$$

where $\mu=\cos (\vartheta)$ is sampled uniformly in the interval $(-1,1)$ as $2 r_{\vartheta}-1$ and $\phi$ is sampled uniformly between 0 and $2 \pi$ as $2 \pi r_{\phi}$, with $r_{\vartheta}$ and $r_{\phi}$ random deviates in the interval $[0,1)$.
[1] P. S. Brantley, C. A. Hagmann, J. A. Rathkopf, MCAPM$C$ Generator and Collision Routine (Gen2000/Bang2000) Documentation, UCRL-MA-141957 (2003).

[2] Which cross section is used in the sampling of points $i$ ) and iii) depends on the model used to sample the velocity of the target and will be explained in the coming sections.

[3] Throughout this report we adopt the convention for which $c=k=1$, where $c$ is the speed of light and $k$ is the Boltzmann constant. Energies, masses and temperatures have the same units.

[4] D. E. Cullen and C. R. Weisbin, Nucl. Sci. Eng. 60, 199 (1976).

[5] G. W. Hedstrom, L. J. Cox and S. T. Perkins, The content and Structure of MCF files, UCRL-ID-127438, Rev. 1 (1997).
[6] X-5 Monte Carlo Team, MCNP A General Monte Carlo N-Particle Transport Code, Version 5, LA-UR-03-1987 (2003).

[7] D. E. Cullen, THERMAL: A Routine Designed to Calculate Neutron Thermal Scattering, UCRL-ID-120560-REV1 (1995).

[8] The neutron-energy cutoff is $E_{\max }=10^{4} \mathrm{~T}$ or $0.01 \mathrm{MeV}$, whichever is smaller, unless $10^{2} T>0.01 \mathrm{MeV}$, in which case $E_{\max }=10^{2} \mathrm{~T}$ or $10 \mathrm{MeV}$, whichever is smaller. Here $E_{\max }$ and $T$ (the temperature of the target) are in units of $\mathrm{MeV}$.

[9] The operation of heating the $T=0$ multi-group cross section smears the group boundaries. Because of this, $\sigma^{*}\left(E_{i}, T\right)$ has to be treated as point-wise data. 